\title{
The Role of the Speech-Language Pathologist in Managing Dysphagia in the Elderly
}

\section{Dennis M. Ruscello*}

Department of Communication and Disorders, West Virginia University, Morgantown, WV, USA

"Corresponding author: Dennis M. Ruscello, 805 Allen Hall, P. O. 6122, West Virginia University, Morgantown, West Virginia, USA, Tel: 304-293-2894; Fax: 304-293-2905; E-mail: Dennis.Ruscello@mail.wvu.edu

Rec date: Mar 17, 2015; Acc date: Apr 27, 2015; Pub date: Apr 30, 2015

Copyright: @ 2015 Ruscello DM. This is an open-access article distributed under the terms of the Creative Commons Attribution License, which permits unrestricted use, distribution, and reproduction in any medium, provided the original author and source are credited.

\begin{abstract}
Dysphagia is a disorder of swallowing and is frequently present in the elderly. A swallowing disorder may be a function of the normal aging process or related to some disease process such as stroke or other medical issues. Patients generally exhibit different symptoms that indicate referral for an evaluation of the swallowing mechanism. The speech-language pathologist (SLP) is responsible for the diagnosis of swallowing problems and determining a course of treatment. There are a number of different treatments that may be used, but selection is a function of the specific problem. There is an evidence base to support the various treatments, but the level of evidence consists primarily of case studies and small cohort studies. The population of elderly is increasing, which means that the number of persons with dysphagia will continue to increase. Appropriate management begins with referral and leads to interdisciplinary management by a team of healthcare professionals. Professionals who work with the elderly should be aware of the potential for dysphagia and be aware of the clinical symptoms, so that an appropriate care plan may be implemented.
\end{abstract}

Keywords: Deglutition; Dysphagia; Evaluation; Treatment; Swallowing

\section{Introduction}

The act of eating or deglutition is generally a pleasurable activity with the primary purpose of providing necessary nutrition and hydration for sustaining life [1].It is conceptualized as an activity that begins under voluntary control and then shifts to involuntary control. Different foods and food textures provide distinct sensory characteristics that stimulate receptors such as touch, temperature, taste and smell. Normal swallowing is a dynamic interaction of finely controlled motor and sensory actions that allow liquids and solids to move from the oral cavity to the stomach very quickly. Physiologically, food intake requires swallowing, which has been characterized as an intricately organized process that is comprised of four stages. The four stages are the oral preparatory, oral, pharyngeal, and esophageal [2].

During the oral preparatory stage, the individual takes in the food and prepares it into a cohesive mass known as a bolus [2]. The amount and type of oral preparation will vary as a function of the viscosity of the food, which can range from a thin liquid to a solid that requires significant chewing to prepare. Once the bolus is prepared, it is transported during the oral stage to the back of the oral cavity proximate to the area of the oropharynx or opening to the throat. When the bolus is propelled toward the area of the oropharynx, the pharyngeal stage swallow response is triggered. At this stage, there are a number of events that occur to protect the airway and propel the bolus into the pharynx or throat. Once the bolus enters the pharynx, there is relaxation of a band of muscle behind the voice box or larynx. This band of muscle known as the upper esophageal sphincter opens to allow bolus passage into the esophagus.

Although swallowing is characterized as a series of discrete events, it is a rapid process. The actual duration of each stage will vary according to the type and volume of food being swallowed but transit times in healthy young adults typically remain in the 1 second or less range each for both oral and pharyngeal stages of swallowing [3-6].In the elderly, there are changes in the anatomy and physiology of the swallowing mechanism that are a function of age and may affect oral feeding. In addition, there is also a population of elderly with swallowing problems due to different medical pathologies [4-6]. A person who experiences problems with swallowing is diagnosed as having dysphagia.

It is important that allied healthcare professionals as well as primary care and specialty physicians understand the normal aging process and disease processes associated with swallowing, so that they have the appropriate knowledge base to identify such patients and initiate referrals, when necessary. They also need to be aware of the role of the speech-language pathologist (SLP) and be cognizant of different assessment and intervention procedures that are utilized for individuals with dysphagia [4-7]. This paper examines changes in swallowing physiology in the normal aging process and in various diseased states. It also reviews the role of SLP in the assessment and treatment of patients. Finally, recommendations for a plan of care are made.

\section{Swallowing in the Elderly Population}

\section{Normal aging process}

Chodzko and Ringel [8] indicate that aging results in alterations occurring at the molecular, cellular and organ levels of the individual. The changes result in a sequential deterioration of the body's capacity to react to changes of homeostatic equilibrium with the end result being a drop in human performance. The structural and behavioral outcomes of aging are fairly uniform across most physiological systems. Features such as atrophy, dystrophy, and edema may be 
noted and often signal morphological changes like reduced elasticity and demyelination. The end result is that aging organ systems show slower response times, and decreases in strength, stability, coordination and stamina. For instance, vision and hearing are two of the more common sensory systems that show decline with age but can be corrected in many cases through the use of corrective lenses or assistive listening devices such as hearing aids.

\section{Swallowing mechanism change with age}

Different authors [1-6] have summarized the effects of aging on the act of swallowing and indicate that healthy individuals 65 and older show changes in swallowing that may be attributed to age alone. There are reported changes in sensation, movement and bone composition. For example, the sensory characteristics of taste, touch, and smell are diminished and can adversely affect food intake. In addition, there is documented loss of oral muscle strength secondary to alterations in muscle mass and contractility. Certain anatomical structures such as the cartilage of the larynx and hyoid bone undergo ossification.

Data $[1,6]$ reported to date indicate that there are corresponding subtle changes in swallowing physiology. For instance, swallow duration or the speed of swallowing a bolus increases slightly with age but remains within the range of normal transit times. The elderly also require additional jaw strokes to prepare foods that require mastication. Chewing is likewise influenced by dental status, since extended chewing times in some patients are often associated with illfitting dentures or an edentulous state. Bolus residue in the oral cavity and pharynx increases slightly with age but is eventually cleared without problem. Research has also found that the elderly cannot maintain lingual isometric tasks at particular levels when compared with younger individuals. Collectively, these changes in anatomy and physiology can have an impact on the community dweller's swallowing skills, particularly if they have a temporary illness or are under conditions of stress [4]. Sura et al. [9] report a 37.6\% prevalence of swallowing problems/complaints in a group of 65-94-year-old community dwelling adults. The authors also indicated that $5.2 \%$ of the respondents reported placement of a feeding tube at some time in life, and $12.9 \%$ reported using nutritional supplements to achieve satisfactory daily caloric intake.

\section{Disease onset dysphagia}

Often times, individuals incur a specific medical problem and a swallowing disorder is part of the medical problem. The etiologic categories of dysphagia in the elderly include neurologic disease, head/ neck cancers, esophageal disorders, respiratory, and iatrogenic disorders [10-12]. Figure 1 presents the general categories and examples within each of the categories. The most frequent causative agents across the categories as reported in the literature are neurologic and oncologic. Reviews of the literature indicate that some patients with nonprogressive brain disease and those with neurodegenerative brain disease present are at risk for malnutrition [9,12].Statistical percentages of affected patients indicated a range of $15 \%$ to $20 \%$ in the different samples that were examined. The presence of malnutrition is associated with higher morbidity and mortality due to a number of factors such as a reduced immune response, diminished capability to recuperate from illness and wound healing, limited respiratory effort and overall muscle strength.

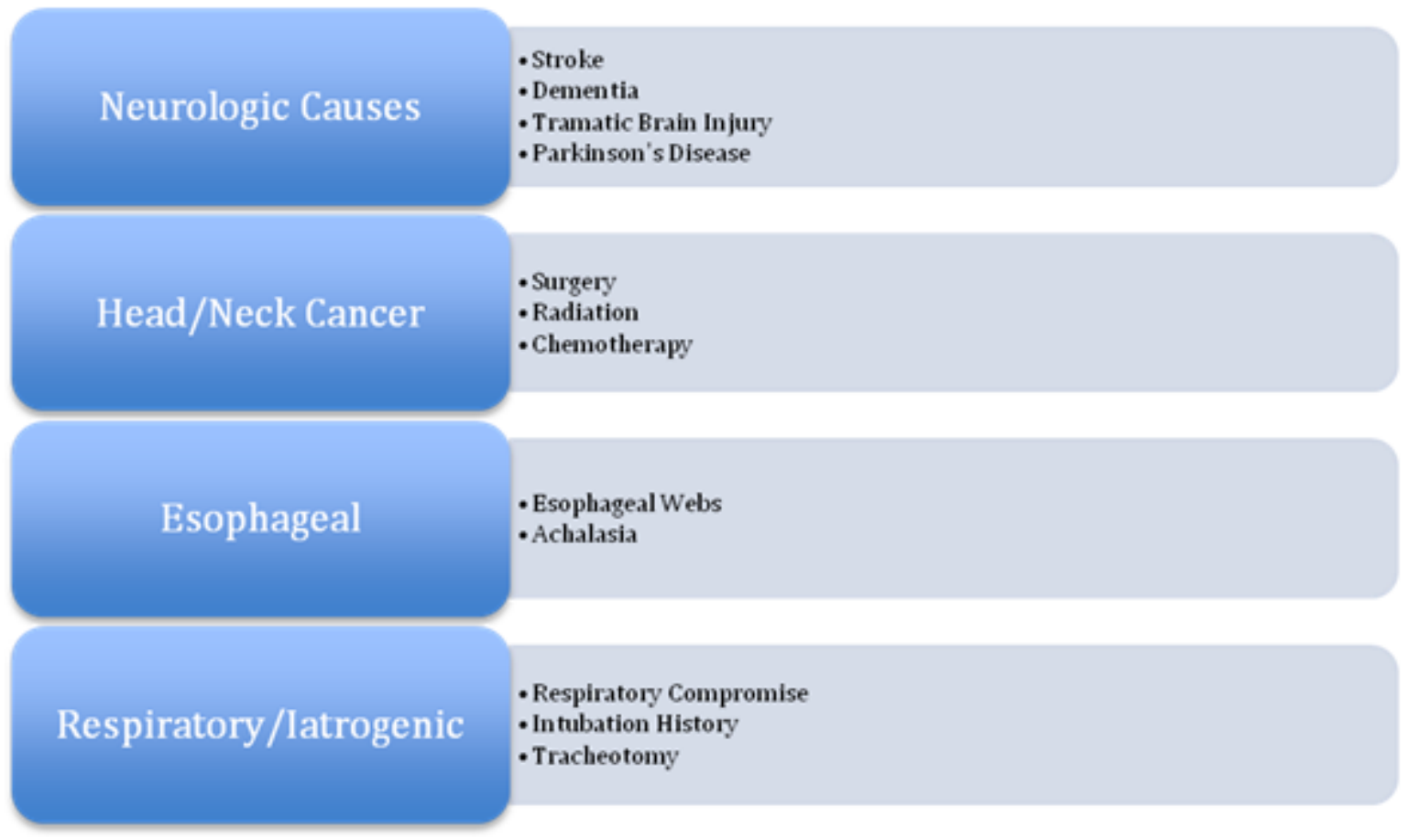

Figure 1: Etiological categories associated with dysphagia. 


\section{Symptoms of swallowing disorders}

When the act of swallowing is disrupted at any stage, the individual presents with dysphagia [4-6].The signs of dysphagia may manifest in certain anatomical areas such as the oral cavity, pharynx, larynx, and/or esophagus [2].The signs or symptoms are indicative of some underlying condition. For example, malnutrition and aspiration pneumonia are frequent symptoms of the elderly diagnosed with dysphagia [9]. While malnutrition and aspiration pneumonia are major symptoms of dysphagia, there are other more subtle problems that should alert healthcare specialists to the possible presence of dysphagia [6]. For instance, patients may report behaviors before or after meals such as choking, coughing and excessive throat clearing, which may be symptomatic as are reports of food "stuck in the throat or chest" (globus) or the avoidance of eating a particular food texture. Reported histories of different problems such as pulmonary infection or weight loss are also potential indicators that may suggest the presence of dysphagia. A list of potential symptoms is summarized in Figure 2.

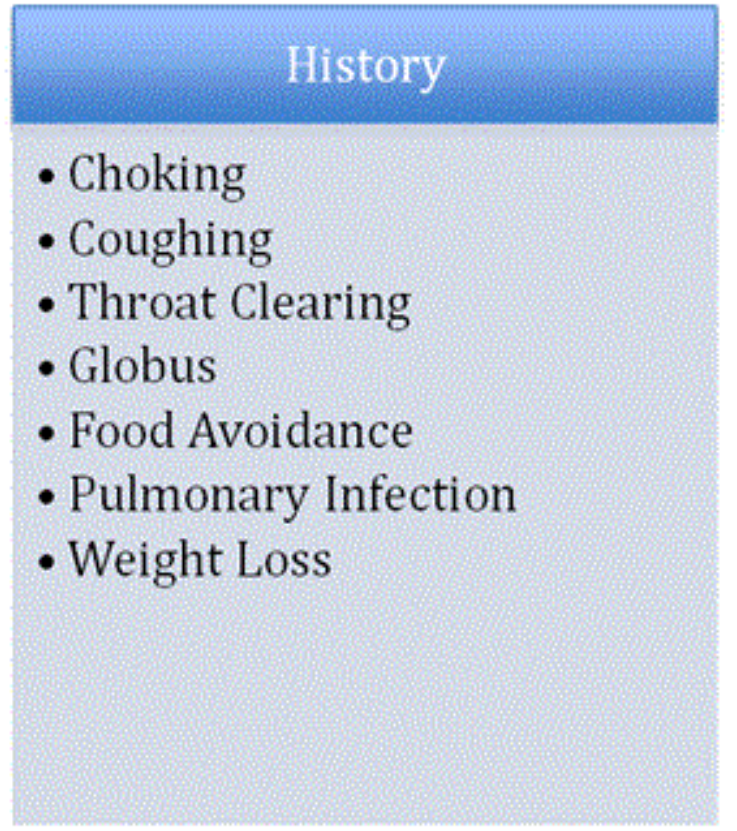

Figure 2: Symptoms that are suggestive of dysphagia.

Clinical observation of a patient while eating is an important component of determining the need for referral. Professionals need to be mindful of the fact that the clinical markers of dysphagia can be somewhat variable across geriatric patients $[12,13]$. A patient may exhibit fluctuating symptoms over time, which suggests repeat observations can be very helpful, if a problem is suspect. Often times, observing the patient at mealtime to monitor swallowing is the initial step in the referral process. There are a number of clinical signs that should alert healthcare professionals to the presence of a swallowing disorder. For instance, significantly extended mealtimes or prolonged chewing, food residue remaining in the oral cavity, nasal reflux of food through the nose, excessive drooling during a meal, and/or changes in quality of the voice such as a gurgly voice quality following a meal are potential signs. Other symptoms may be harder to associate with dysphagia like observable changes in respiration, food refusal or limited food intake, and avoidance of certain food textures. Note that the symptoms discussed are suggestive of dysphagia and indicate the need for a referral $[4,5]$.The SLP is the healthcare professional who possesses the knowledge and skills necessary to diagnose and treat individuals with dysphagia [4,7].SLPs are generally part of a healthcare team employed in hospitals, skilled nursing facilities, rehabilitation settings and various community-based clinics and home health organizations.

\section{Assessment of swallowing disorders}

Generally, the first step in the assessment process for the SLP is to conduct a clinical examination of swallowing, which is also known as a bedside examination [6].It is a screening process that allows the collection of patient performance data. The main components of this assessment are the collection of a patient's medical history, an assessment of the structure and function of the swallowing mechanism, and observing the patient's swallowing with different food viscosities and quantities. Logemann [6] states that the clinical examination provides information that enables the SLP to answer certain questions and identify the need for further testing. The pertinent diagnostic information includes: (1) recognizing the causal factor(s) that explains the swallowing disorder, (2) developing a tentative hypothesis that describes the swallowing disorder, (3) formulating a provisional treatment plan, (4) identifying any issues that would indicate additional testing, and (5) determining if the patient can tolerate further testing. The clinical examination furnishes important information and in some cases, the SLP can clear a patient 
for oral feeding, recommend modifications in the diet, and/or recommend a particular treatment.

In other cases, the performance of the patient during the screening indicates the need for more comprehensive testing, since some problems such as silent aspiration cannot be detected. Instrumental testing allows direct observation of the anatomy and physiology of those structures that are involved in the swallowing process $[2,3]$. In particular, observation of functional elements such as the pharyngeal phase and esophageal stages of swallowing cannot be observed during the clinical examination. The most commonly used instrumental procedures are video fluorography and flexible endoscopy. Video fluorography also known as a modified barium swallow study (MBSS) provides a video X-ray image of the upper digestive tract, while a patient is ingesting different quantities of various food textures that have been placed in a barium contrast. This test enables the SLP to assess the patient's swallow physiology and identify any problems that may present with the swallow. Instrumental testing also permits the SLP to assess compensatory feeding strategies and make recommendations for treatment. The flexible endoscopy procedure is generally referred to as fiber optic endoscopic evaluation of swallowing (FEES) and involves passing an endoscope via the naris into the nasopharynx. The SLP positions the scope and observes the patient's swallowing.

Each of the instrumental procedures has advantages and disadvantages [5]. The MBSS furnishes the examiner with a more complete picture of swallowing anatomy/physiology that may include structures from the lips to the stomach, while FEES allows visualization of the pharynx (throat) only. The entire swallowing event is available through the MBSS but is limited with FEES. The SLP can observe events in the pharynx before and after the swallow, but cannot observe the actual moment of swallowing due to reflection of the scope, which is known as the "whiteout" effect. If a patient is aspirating some food consistency, it can be seen during the swallow when undergoing an MBSS, but it cannot with FEES. The examiner would, however, be able to observe aspiration before or after the swallow with the FEES procedure. It is also to be noted that FEES is more portable and can be used with patients who are unable to tolerate placement in the radiation suite. Finally, the MBSS is a procedure that utilizes radiation, thus limiting the duration of the procedure; however, FEES can be used on a more frequent basis without the risk of radiation over exposure.

\section{Treatment of swallowing disorders}

When a patient is diagnosed with a swallowing disorder, treatment is designed to improve food flow and/or swallowing physiology, so that the patient may receive adequate oral nutrition and hydration to sustain life [9].The SLP summarizes the results of the assessment and makes recommendations to the patient's physician who will then approve a course of action. Initially, some patients may receive nutrition and hydration through some type of non oral means like a nasogastric tube or a gastrostomy. If swallowing skills improve, the patient can then be transitioned to oral feeding; however, in some cases the patient does not recover adequate function and is fed primarily through non oral means. When swallowing treatment is recommended, the SLP may employ different treatments that include: (1) compensatory strategies, (2) sensory stimulation techniques, (3) swallowing maneuvers, and (4) direct therapy exercise with or without supplementary techniques [4-6].

\section{Compensatory strategies}

Compensatory strategies are techniques that are used to manage food flow and minimize the patient's symptoms such as the aspiration of food. Compensatory strategies do not restore function but rather assist the person in creating a safer feeding environment [13].There are a number of different strategies that include: (1) changes in head or body posture that alter the dimensions of the pharynx thus changing food flow; (2) adjusting the amount of food and pacing the presentation of food to the patient; (3) modifying the thickness or thinness of food; and (4) introducing some type of fabricated oral prosthesis.

Some patients can benefit from the use of different postural techniques $[13,14]$.For instance, the chin down or chin tuck position is used very frequently. Food is introduced and the patient then moves the head down with the chin in contact with the neck during a swallow. This position is useful for a number of different problems, because it acts to change the relationships among the different pharyngeal structures. It is also very easy for the patient to learn with only a minimum of verbal cueing and practice needed. Postural strategies are often used on a short-term basis, because some patients regain function, while others benefit from direct therapy procedures and use the postural techniques in transition.

Adjusting the amount or volume of food per swallow can benefit some individuals with swallowing disorders [15].A larger or smaller bolus may be of benefit, because it may facilitate a more effectual swallow. Similarly, pacing can be beneficial for some patients. For instance, slowing down food intake can help a patient better prepare the food for swallowing and also assist in clearing the food after the swallow has triggered. The use of certain food consistencies and the elimination of others are used very frequently. According to Logemann, [6] food consistency changes should be used as a last resort, because many patients experience problems when certain food consistencies are eliminated. That is, the sensory change in a food or certain foods is not appealing to many patients; consequently, there is poor patient compliance. For example, thickened water is a sensory experience that is not very appealing to some who experience problems with thin liquids. Finally, prosthetic devices can be constructed and used to improve swallowing skills [16].These are oralbased appliances that are fabricated by a prosthodontist. Prostheses are used with patients who have oral tissue loss due to different cancers or present with significant neurological deficits of the tongue or soft palate.

\section{Sensory stimulation techniques}

Enhancement techniques are used to heighten incoming sensory information and facilitate a more efficient swallow [6].They can be incorporated into actual eating contexts or used as adjunct techniques to improve swallowing skills. For instance, some very basic therapeutic techniques include placing downward pressure on a spoon when introducing a bolus and/or altering the bolus along the dimensions of taste, temperature, viscosity and volume. Another frequently employed enhancement technique is thermal tactile stimulation $[17,18]$.This treatment technique involves stimulating the posterior portion of the oral cavity and back of the throat with a laryngeal mirror or swab that has been chilled in order to improve the pharyngeal swallow. 


\section{Swallow maneuvers}

Swallow maneuvers are variations of normal swallowing that are designed to assist in creating an effective and safe swallow $[19,20]$.They are frequently employed on a short-term basis and gradually eliminated as the patient's swallowing skills improve. Maneuvers include the supraglottic, and super supraglottic swallows the Mendelsohn procedure and the effortful or hard swallow. Each addresses a different aspect of swallow dysfunction. For instance, the supraglottic and super supraglottic swallows require patients to hold their breath before saliva swallows or food intake. The maneuvers act to facilitate closure of the vocal folds. The implication is that the breath holding maneuvers will aid in preventing the aspiration of food into the lungs. The Mendelsohn is utilized to improve function of the upper esophageal sphincter, while the effortful swallow is used to enhance swallow force thus improving the clearance of food and minimizing residue in the pharynx.

\section{Direct exercise programs}

Direct exercise programs aim to improve swallow physiology through some form of exercise that focuses of a specific swallow subsystem or general swallow function [5,6].There are a number of different techniques or programs that use muscle-training regimens. Some of those reported in the literature are lingual resistance, Masako, Shaker/head-lift, EMST (expiratory muscle strength training), and MDTP (McNeill dysphagia therapy program) [21-27].Lingual resistance training introduces exercise to increase tongue strength through an exercise known as a tongue press. The patient is instructed to press the tongue against the roof of mouth with the repetitions and pressure of the tongue in contact with the roof of the mouth varied. This is a type of isometric exercise that is used to build strength via muscular tension. Physiologically, isometrics involve muscle exercises that do not change the length of a specific muscle or muscle group. The results of several studies suggest that tongue strength can be improved with a corresponding improvement in swallowing skills $[21,22]$. The Masako or tongue holding technique is used in exercise programs to improve strength and range of motion of the glossopharyngeus, which is a portion of the superior constrictor muscle [23].

The Shaker/head lift is an exercise program for improving laryngeal elevation and upper esophageal sphincter function (UES) by strengthening the muscle groups that are involved in UES function [24].The patient lies on her/his back and raises the head using both exercise repetition and sustained elevation to improve muscular strength. The EMST program was developed to strengthen the respiratory muscles of expiration, and a review conducted by Laciuga et al. [25] indicates that there is some preliminary data to indicate parallel changes in swallowing skills for some patients. Finally the MDTP is an example of an exercise program that uses exercise principles of task specificity, resistive load, speed, and variability $[26,27]$. During treatment sessions, subjects are taught to practice hard swallows under different training conditions that are modified as a function of bolus consistency and volume, eating rate, and amount of oral intake. The goals of the program are to build strength and coordination that will lead to normalization of swallowing skills.

In addition to the exercise programs discussed, there are supplemental treatment adaptations that are used in conjunction with exercise. The optional adaptations most frequently used are NMES (neuromuscular electrical stimulation) and biofeedback. NMES delivers electrical stimulation to muscles affected by the specific swallowing disorder provided that peripheral nerve function is intact [28-30].The stimulation is delivered to the muscles of the head and neck via surface electrodes, while the patient is engaged in swallowing exercises. The rationale is that electrical stimulation will enhance the contraction of muscles that were ineffectual due to the swallowing disorder. The typical treatment has the patient practice swallowing with the stimulation and then carrying out home practice with or without the device. An additional supplementary technique that is used consists of different forms of biofeedback. Biofeedback has a long and significant history in our profession for the treatment of a variety of speech disorders and has now been used in swallowing rehabilitation. The patient is given a physiological signal that is generally not available during conscious execution of a skill [31].The biofeedback technique of sEMG has been used with patients. They receive an electrical signal of neuromuscular activity that is detected during swallowing $[32,33]$. The information is used by the learner to modify performance in a positive way.

\section{Discussion}

It is obvious that deglutition is critical to sustaining life because it is the means for satisfying our nutrition and hydration needs. In addition, eating is generally a pleasurable social activity that is enjoyed by most people. It is clear from this discussion that normal swallowing is a complex process that has been discussed in terms of stages beginning when food is placed in the mouth and ends in the stomach. There is a body of research that indicates swallow physiology changes with age due to a number of different factors. These changes are observed in sensory, motor, and structural body systems that support swallowing. These changes can leave some elderly vulnerable to swallowing problems. For instance, Barczi et al. [34] indicated that diminished oropharyngeal functional reserve is part of the aging process. It can often put the elderly at greater risk for dysphagia, since there is a higher likelihood that environmental stressors such as chronic illness or other medical conditions stress their functional capacity, thus giving rise to a swallowing problem. Healthcare professionals should be alert to symptoms that were discussed herein. Training sessions such as informational presentations by an SLP can also assist professionals who provide services to community dwellers in forming an awareness of potential swallowing problems.

For example, Daniels and her associates [35] developed a screening protocol that they used to identify patients who may need instrumental testing such as video fluorography. Patients were required to drink calibrated volumes of water and were evaluated for dysphonia, dysarthria, abnormal volitional cough, abnormal gag reflex, cough following a swallow, and voice change following a swallow. If a patient demonstrated at least two of the six behavioral predictors, she/he was referred for an instrumental study. An SLP could use the list of predictors and develop an instructional module that could be used to educate various professional groups and the elderly. If such symptoms were noted in an actual situation, the healthcare professional could discuss the problem with the individual and seek their permission for referral.

Elderly patients who develop swallowing disorders due to some medical condition such as stroke or head/neck cancer are generally managed by a healthcare team that includes an SLP [4-6].Team composition may vary but often consists of a dietician, nurse, occupational therapist, physical therapist, physicians which may include an otolaryngologist, gastroenterologist, neurologist and/or 
Page 6 of 7

radiologist, respiratory therapist and social worker. Each professional provides a unique perspective in the development of a rehabilitation program for the patient. Collectively, they formulate an interdisciplinary rehabilitation plan to minimize the impact of the swallowing disorder and any additional problems that manifest. For instance, the SLP may work with the dietician to create an appropriate diet for the patient. The dietician determines the patient's nutritional and hydration needs, while the SLP determines the safest way to provide the nutrition and hydration. This would be in supplement to treatment for the swallowing disorder. In addition, the SLP may work with the occupational and physical therapists if corrective patient positioning is needed to improve feeding performance or transfer of food from hand to mouth is a problem. Such interdisciplinary cooperation ensures that the patient receives sufficient nutrition and hydration and treatment directed to maximum recovery of all physiological functions [36].

Finally, it is evident that the SLP has a number of different therapeutic interventions available for use. The selection of a specific intervention will depend on the patient's swallowing disorder and the severity of the disorder; however, an important issue that must be considered is the evidence that is available to support the different treatments that $[36,37]$. What scientific evidence is available to either support or refute the different treatments? In most cases, there is some evidence from case studies or small cohort studies to support the efficacy of most treatments; however, definitive randomized controlled trials are lacking. An additional caveat is that research findings for some of the treatments are equivocal. For example, thermal stimulation is used quite frequently to stimulate initiation of the pharyngeal swallow, but there are studies that both support and refute the treatment $[17,18]$. Similarly, NMES is a treatment that is used very frequently, but it is very controversial, because current research findings are equivocal [5]. Readers are referred to Practice Portal of the American Speech-Language-Hearing Association (http://ncepmaps.org/ adultdysp/) for current evidence reviews regarding the different treatments that have been discussed [37].

\section{Summary}

Dysphagia among the elderly is a significant problem with estimates of between 300,00 and 600,000 new cases per year and health care statistics showing that between $30 \%$ and $40 \%$ of nursing home residents have dysphagia $[34,38]$. It is likely that numbers of elderly with dysphagia will increase, since the elderly population is increasing and more people with chronic illnesses are living longer. The clinical care of older dysphagic adults requires a comprehensive assessment of variables that consist of standard risk factors, physiological indices, and clinical concerns. The assessment needs to be tailored to the unique characteristics and limitations of older adults and be adaptable across a continuum of care settings. An inclusive care model that incorporates a patient-centered approach with a strong emphasis on patient choice if possible, quality of life, and functional status is recommended as the most appropriate to meet the needs of the elderly $[34,39]$. Healthcare professionals need to be cognizant of the signs of dysphagia in community dwellers and those in different care settings. Early identification and referral are the first steps in the process of assisting the elderly patient who may present with dysphagia.

\section{References}

1. Gleeson DC (1999) Oropharyngeal swallowing and aging: a review. J CommunDisord 32: 373-395.
2. Corbin-Lewis K, Liss JM, Sciortino, KL (2005) Clinical anatomy and physiology of the swallow mechanism. Clifton Park, Thomson Delmar Learning.

3. Shaw SM, Martino R (2013) The normal swallow: muscular and neurophysiological control. OtolaryngolClin North Am 46: 937-956.

4. Groher ME (1997) Dysphagia: diagnosis and management (3rded). Butterworth-Heinemann, Boston.

5. Groher ME, Crary MA (2010) Dysphagia: clinical management in adults and children. St. Louis, Mosby.

6. Logemann, JA (1998) Evaluation and treatment of swallowing disorders. Austin, PROED.

7. American Speech-Language-Hearing Association (2001) Roles of speechlanguage pathologists in swallowing and feeding disorders: technical report [Technical Report]. Available from www.asha.org/policy.

8. Chodzko-Zajko WJ, Ringel RL (1987) Physiological aspects of aging. Journal of Voice 1: 18-26.

9. Sura L, Madhavan A, Carnaby G, Crary MA (2012) Dysphagia in the elderly: management and nutritional considerations. ClinInterv Aging 7: 287-298.

10. Roden DF, Altman KW (2013) Causes of dysphagia among different age groups: a systematic review of the literature. OtolaryngolClin North Am 46: 965-987.

11. Khan A, Carmona R, Traube M (2014) Dysphagia in the elderly. ClinGeriatr Med 30: 43-53.

12. Forster A, Samaras N, Gold G, Samaras, D (2011) Oropharyngeal dysphagia in older adults: a review. Geriatric Medicine 2: 356-362.

13. Logemann JA (1993) Manual for the videofluoroscopic study of swallowing (2nded). Austin, PROED

14. Logemann JA, Rademaker AW, Pauloski BR, Kahrilas PJ (1994) Effects of postural change on aspiration in head and neck surgical patients. Otolaryngol Head Neck Surg 110: 222-227.

15. Lazarus CL, Logemann JA, Rademaker AW, Kahrilas PJ, Pajak T, et al. (1993) Effects of bolus volume, viscosity, and repeated swallows in nonstroke subjects and stroke patients. Arch Phys Med Rehabil 74: 1066-1070.

16. Davis JW, Lazarus C, Logemann J, Hurst PS (1987) Effect of a maxillary glossectomy prosthesis on articulation and swallowing. J Prosthet Dent 57: 715-719.

17. Rosenbek JC, Roecker EB, Wood JL, Robbins J (1996) Thermal application reduces the duration of stage transition in dysphagia after stroke. Dysphagia 11: 225-233.

18. Rosenbek JC, Robbins J, Fishback B, Levine RL (1991) Effects of thermal application on dysphagia after stroke. J Speech Hear Res 34: 1257-1268.

19. Lazarus CL (1993) Effects of radiation therapy and voluntary maneuvers on swallow functioning in head and neck cancer patients. ClinCommunDisord 3: 11-20.

20. Martin BJ, Logemann JA, Shaker R, Dodds WJ (1993) Normal laryngeal valving patterns during three breath-hold maneuvers: a pilot investigation. Dysphagia 8: 11-20.

21. Robbins J, Gangon RE, Theis SM, Kays SA, Hewitt AL, et al. (2005) The effects of lingual exercise on swallowing in older adults. J Am GeriatrSoc 53: 1483-1489.

22. Robbins J, Kays SA, Gangnon RE, Hind JA, Hewitt AL, et al. (2007) The effects of lingual exercise in stroke patients with dysphagia. Arch Phys Med Rehabil 88: 150-158.

23. Fujiu M, Logemann, JA (1996) Effect of a tongue-holding maneuver on posterior pharyngeal wall movement during deglutition. American Journal of Speech-Language Pathology 5: 23-30.

24. Logemann JA, Rademaker A, Pauloski BR, Kelly A, Stangl-McBreen C, et al. (2009) A randomized study comparing the Shaker exercise with traditional therapy: a preliminary study. Dysphagia 24: 403-411.

25. Laciuga H, Rosenbek JC, Davenport PW, Sapienza CM (2014) Functional outcomes associated with expiratory muscle strength training: narrative review. J Rehabil Res Dev 51: 535-546. 
Citation: Ruscello DM (2015) The Role of the Speech-Language Pathologist in Managing Dysphagia in the Elderly. J Gerontol Geriatr Res 4: 213. doi:10.4172/2167-7182.1000213

Page 7 of 7

26. Carnaby-Mann GD, Crary MA (2010) McNeill dysphagia therapy program: a case-control study. Arch Phys Med Rehabil 91: 743-749.

27. Crary MA, Carnaby GD, LaGorio LA, Carvajal PJ (2012) Functional and physiological outcomes from an exercise-based dysphagia therapy: a pilot investigation of the McNeill Dysphagia Therapy Program. Arch Phys Med Rehabil 93: 1173-1178.

28. Kiger M, Brown CS, Watkins L (2006) Dysphagia management: an analysis of patient outcomes using VitalStim therapy compared to traditional swallow therapy. Dysphagia 21: 243-253.

29. Carnaby-Mann GD, Crary MA (2008) Adjunctive neuromuscular electrical stimulation for treatment-refractory dysphagia. Ann OtolRhinolLaryngol 117: 279-287.

30. Bülow M, Speyer R, Baijens L, Woisard V, Ekberg O (2008) Neuromuscular electrical stimulation (NMES) in stroke patients with oral and pharyngeal dysfunction. Dysphagia 23: 302-309.

31. Bryant M (1991) Biofeedback in the treatment of a selected dysphagic patient. Dysphagia 6: 140-144.

32. Crary MA, Carnaby Mann GD, Groher ME, Helseth E (2004) Functional benefits of dysphagia therapy using adjunctive sEMG biofeedback. Dysphagia 19: 160-164.
33. Chen YW, Chang KH, Chen HC, Liang WM, Wang YH, et al. (2015) The effects of surface neuromuscular electrical stimulation on post-stroke dysphagia: A systemic review and meta-analysis. ClinRehabil.

34. Barczi SR, Sullivan PA, Robbins J (2000) How should dysphagia care of older adults differ? Establishing optimal practice patterns. Semin Speech Lang 21: 347-361.

35. Mehta RJ (1975) Pyridine nucleotide-linked oxidation of methanol in methanol-assimilating yeasts. J Bacteriol 124: 1165-1167.

36. Daniels SK, Ballo LA, Mahoney MC, Foundas AL (2000) Clinical predictors of dysphagia and aspiration risk: outcome measures in acute stroke patients. Arch Phys Med Rehabil 81: 1030-1033.

37. Geeganage C, Beavan J, Ellender S, Bath PM (2012) Interventions for dysphagia and nutritional support in acute and subacute stroke. Cochrane Database Syst Rev 10: CD000323.

38. Palmer RM, Landefeld CS, Kresevic D, Kowal J (1994) A medical unit for the acute care of the elderly. J Am GeriatrSoc 42: 545-552.

39. Machin J, Shaw C (1998) A multidisciplinary approach to head and neck cancer. Eur J Cancer Care (Engl) 7: 93-96. 